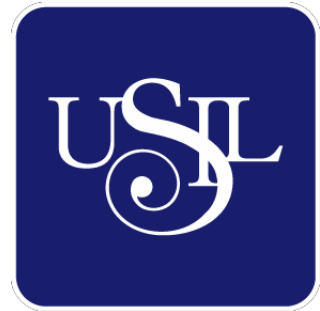

UNIVERSIDAD

SAN IGNACIO

DE LOYOLA

FACULTAD DE INGENIERÍA

Carrera de Ingeniería Industrial

\title{
DISEÑO DE SISTEMA CONTRA INCENDIOS DE AGUA NEBULIZADA PARA REDUCIR LAS PÉRDIDAS EN INFRAESTRUCTURA DE PROCESADORA AGRÍCOLA
}

Tesis para optar el Título Profesional de Ingeniero Industrial

\author{
HOMERO LIZA YUNQUE \\ JUAN RAMON PIZARRO QUISPE
}

Asesor:

Mg. Ing. Pedro Pablo Rosales Lopez

Lima - Perú

2019 
JURADO DE LA SUSTENTACIÓN ORAL

Presidente

Jurado 1

Jurado 2

Entregado el:

Aprobado por:

Graduandos:

Homero Liza Yunque

Juan Ramon Pizarro Quispe
Asesor de Tesis:

Pedro Pablo Rosales López

Pedro Pablo Rosales López 


\section{DECLARACIÓN DE AUTENTICIDAD}

Yo, Homero Liza Yunque, Identificado con DNI N. ${ }^{\circ} 41649783$, Bachiller del Programa Académico de la Carrera de Ingeniería Industrial, de la Facultad de Ingeniería de la Universidad San Ignacio de Loyola, presento la tesis titulada: “DISEÑO DE SISTEMA CONTRA INCENDIOS DE AGUA NEBULIZADA PARA REDUCIR LAS PÉRDIDAS EN INFRAESTRUCTURA DE PROCESADORA AGRÍCOLA".

Declaro en honor a la verdad, que el trabajo de tesis es de mí autoría; que los datos, los resultados y su análisis e interpretación, constituyen a mí aporte.

Todas las referencias han sido debidamente consultadas y reconocidas en la investigación.

En tal sentido, asumo la responsabilidad que corresponda ante cualquier falsedad $u$ ocultamiento de la información aportada. Por todas las afirmaciones, ratifico lo expresado, a través de mi firma correspondiente.

Lima, 07 de febrero de 2019

Homero Liza Yunque

DNI N. ${ }^{\circ} 41649783$ 


\section{DECLARACIÓN DE AUTENTICIDAD}

Yo, Juan Ramon Pizarro Quispe, Identificado con DNI N. ${ }^{\circ}$ 10231279, Bachiller del Programa Académico de la Carrera de Ingeniería Industrial, de la Facultad de Ingeniería de la Universidad San Ignacio de Loyola, presento la tesis titulada: “DISEÑO DE SISTEMA CONTRA INCENDIOS DE AGUA NEBULIZADA PARA REDUCIR LAS PÉRDIDAS EN INFRAESTRUCTURA DE PROCESADORA AGRÍCOLA".

Declaro en honor a la verdad, que el trabajo de tesis es de mí autoría; que los datos, los resultados y su análisis e interpretación, constituyen a mí aporte.

Todas las referencias han sido debidamente consultadas y reconocidas en la investigación.

En tal sentido, asumo la responsabilidad que corresponda ante cualquier falsedad $\mathrm{u}$ ocultamiento de la información aportada. Por todas las afirmaciones, ratifico lo expresado, a través de mi firma correspondiente.

Lima, 07 de febrero de 2019

Juan Ramón Pizarro Quispe

DNI N. ${ }^{\circ} 10231279$ 
EPIGRAFE

Para empezar un gran proyecto,

Solo hace falta, la valentía. Para

Terminar un gran proyecto, hace

Falta perseverancia. (Anónimo). 
DEDICATORIA

Este trabajo, se lo dedicamos a

Nuestros familiares, ya que ellos

Fueron la motivación de nuestro

Desarrollo como profesionales. 


\section{ÍNDICE DE CONTENIDOS}

Pág.

RESUMEN

$\begin{array}{ll}\text { ABSTRACT } & 2\end{array}$

PROBLEMA DE INVESTIGACIÓN 3

IDENTIFICACIÓN DEL PROBLEMA 3

FORMULACIÓN DEL PROBLEMA

$\begin{array}{ll}\text { PROBLEMA GENERAL } & 6\end{array}$

$\begin{array}{lr}\text { PROBLEMAS ESPECÍFICOS } & 6\end{array}$

$\begin{array}{ll}\text { MARCO REFERENCIAL } & 7\end{array}$

$\begin{array}{ll}\text { ANTECEDENTES DE TESIS NACIONALES } & 7\end{array}$

ANTECEDENTES DE TESIS INTERNACIONALES 9

$\begin{array}{ll}\text { ESTADO DEL ARTE } & 13\end{array}$

$\begin{array}{ll}\text { MARCO TEÓRICO } & 16\end{array}$

$\begin{array}{ll}\text { Diagrama de Flujo de Procesos de Procesadora Agrícola } & 16\end{array}$

$\begin{array}{ll}\text { SISTEMAS DE EXTINCIÓN } & 29\end{array}$

$\begin{array}{ll}\text { TIPOS DE EXTINCIÓN } & 30\end{array}$

OBJETIVO DE LA INVESTIGACIÓN 36

OBJETIVO GENERAL 36

OBJETIVOS ESPECÍFICOS 36 
JUSTIFICACIÓN DE LA INVESTIGACIÓN

JUSTIFICACIÓN PRÁCTICA

JUSTIFICACIÓN SOCIAL

JUSTIFICACIÓN ECONÓMICA

JUSTIFICACIÓN AMBIENTAL

HIPÓTESIS DE LA INVESTIGACIÓN

HIPÓTESIS GENERAL

HIPÓTESIS ESPECÍFICAS

ALCANCES DE LA INVESTIGACIÓN

LIMITACIONES DE LA INVESTIGACIÓN

MATRIZ DE CONSISTENCIA

MARCO METODOLÓGICO

METODOLOGÍA

PARADIGMA

ENFOQUE

MÉTODO

VARIABLES

VARIABLE INDEPENDIENTE 
POBLACIÓN Y MUESTRA

POBLACIÓN 46

MUESTRA 46

UNIDAD DE ANÁLISIS 46

INSTRUMENTOS Y TÉCNICAS

INSTRUMENTOS

TÉCNICAS 48

PROCEDIMIENTOS 49

MÉTODO DE ANÁLISIS 52

RESULTADOS 69

DISCUSIÓN 70

CONCLUSIONES 71

RECOMENDACIONES 72

SUGERENCIAS 73

REFERENCIAS

ANEXOS

FORMATO DE INSTRUMENTOS 81 


\section{INDICE DE ILUSTRACIONES}

Pág.

ILUSTRACIÓN 1 INCENDIOS POR AÑO 4

ILUSTRACIÓN 2 MAPA MENTAL DEL SCI DE AGUA NEBULIZADA 13

ILUSTRACIÓN 3. SIMBOLOGÍA DE PROCESOS 16

ILUSTRACIÓN 4. DIAGRAMA DE FLUJO DEL PROCESO 17

ILUSTRACIÓN 5 TRIANGULO DEL FUEGO 22

ILUSTRACIÓN 6 TETRAEDRO DEL FUEGO 24

ILUSTRACIÓN 7 SISTEMA DE CONTROL ELECTRÓNICO 28

ILUSTRACIÓN 8 ROCIADOR DE AGUA

ILUSTRACIÓN 9 INYECTOR DE AGUA 33

ILUSTRACIÓN 10 PULVERIZADOR DE AGUA 33

ILUSTRACIÓN 11 AGENTES GASEOSO DE EXTINCIÓN 34

ILUSTRACIÓN 12 AGENTE QUÍMICO DE EXTINCIÓN 35

ILUSTRACIÓN 13 TIPOS DE AGENTES 35

ILUSTRACIÓN 14 CRONÓMETRO DIGITAL 50

ILUSTRACIÓN 15 ROCIADOR DE AGUA 51

ILUSTRACIÓN 16 PULVERIZADOR DE AGUA 51

ILUSTRACIÓN 17 SCI AGUA NEBULIZADA 53

ILUSTRACIÓN 18 TIEMPO DE EXTINCIÓN

ILUSTRACIÓN 19 ÁREA DE DISTRIBUCIÓN 59

ILUSTRACIÓN 20 CONSUMO DE AGUA

ILUSTRACIÓN 21 PRESUPUESTO 


\section{INDICE DE TABLAS}

Pág.

TABLA 1 MATRIZ DE CONSISTENCIA 42

TABLA 2 VARIABLE INDEPENDIENTE 44

TABLA 3. VARIABLE DEPENDIENTE 45

TABLA 4. MUESTREO DE ÁREAS 55

TABLA 5. MUESTREO TIEMPOS 56

TABLA 6 ÁREA DE DISTRIBUCIÓN 58

TABLA 7 CONSUMO DE AGUA 60

TABLA 8 PRESUPUESTO DE INSTALACIÓN 62

TABLA 9 PRUEBAS DE NORMALIDAD 64

TABLA 10 TIEMPO DE EXTINCIÓN 65

TABLA 11 IGUALDAD DE MEDIAS 65

TABLA 12 ÁREA DE DISTRIBUCIÓN 66

TABLA 13 ÁREA DE DISTRIBUCIÓN 66

TABLA 14 CONSUMO DE AGUA 67

TABLA 15 CONSUMO DE AGUA 67

TABLA 16 PRESUPUESTO DE INSTALACIÓN 68

TABLA 17 TIEMPO DE EXTINCIÓN 68 


\section{INDICE DE ANEXOS}

Pág.

ANEXO I EMPRESA PROCESADORA AGRÍCOLA

ANEXO II EMPRESA PROCESADORA AGRÍCOLA

ANEXO III PLANO DE PLANTA PROCESADORA AGRICOLA

ANEXO IV MÓDULOS DE PRUEBA

ANEXO V SISTEMA NUEVO

ANEXO VI EXTINCIÓN CON NUEVO SISTEMA

ANEXO VII HOJA DE INSTRUMENTOS

ANEXO VIII HOJA TOMA DE DATOS

ANEXO IX PRESUPUESTO SISTEMA ANTIGUO

ANEXO X PRESUPUESTO SISTEMA NUEVO

ANEXO XI PRESUPUESTO SISTEMA NUEVO

ANEXO XII PRESUPUESTO SISTEMA NUEVO

ANEXO XIII HOJA TÉCNICA BOMBA DE SPRINKLER

ANEXO XIV HOJA TÉCNICA TUBERÍAS 88

ANEXO XV HOJA TÉCNICA VÁLVULAS

89

ANEXO XVI HOJA TÉCNICA ROCIADORES

90 


\section{RESUMEN}

En la actualidad en nuestro país, existen muchas pérdidas materiales por la presencia de los incendios, ya que no se cumplen estrictamente, con las normas de seguridad contra incendios en edificaciones; siendo el Reglamento Nacional de Edificaciones (RNE), el documento, que rige las normas de seguridad para todo tipo de edificación, el cual viene siendo modificado año tras año.

Es por eso, que se desea reducir los posibles daños materiales, dentro de una empresa de procesamiento agrícola, ante un posible incendio, que no cuenta con un sistema de control y extinción del fuego, para poder proteger los bienes materiales, y brindar la seguridad a las personas dentro de la empresa.

Observando esta problemática, se diseñó un sistema de protección contra incendios, mediante el sistema de agua nebulizada, que cumple con las normativas y estándares requeridos por el mismo, para así, poder reducir las pérdidas materiales en la infraestructura de la empresa de procesamiento agrícola.

Este diseño contiene un sistema de detección electrónica, y a la vez un sistema de extinción por agua nebulizada, que conjuntamente, dan como resultado la reducción de tragedias, o pérdidas irrecuperables, dando un nivel de seguridad dentro de la empresa.

Para este diseño se aplicó metodología de ingeniería, la cual puede ser aplicable a cualquier tipo de entidad pública o privada y además puede ser auditada, supervisada e inspeccionada por las autoridades competentes. A su vez el diseño de este sistema contra incendios, fue guiado según los estándares de la Asociación Nacional de Protección Contra el Fuego (NFPA), que en su norma 750, establece los criterios mínimos para la utilización de agua nebulizada y su instalación.

\section{Palabras Claves:}

Agua Nebulizada, Pérdidas en Infraestructura y Sistemas Contra incendios. 


\begin{abstract}
At present in our country, there are many material losses due to the presence of fires, since they do not strictly comply with the norms of fire safety in buildings; being the National Building Regulation (RNE), the document, which governs the safety standards for all types of buildings, which is being modified year after year.
\end{abstract}

That is why, it is desired to reduce the possible material damages, within an agricultural processing company, before a possible fire, which does not have a fire control and extinguishing system, to be able to protect the material goods, and to provide security to the people within the company.

Observing this problem, a fire protection system was designed, using the nebulized water system, which complies with the regulations and standards required by it, in order to reduce material losses in the infrastructure of the agricultural processing company.

This design contains an electronic detection system, and at the same time a water mist extinguishing system, which together, result in the reduction of tragedies, or irrecoverable losses, giving a level of security within the company.

For this design engineering methodology was applied, which can be applicable to any type of public or private entity and can also be audited, supervised and inspected by the competent authorities. At the same time, the design of this fire system was guided according to the standards of the National Fire Protection Association (NFPA), which in its 750 standard, establishes the minimum criteria for the use of water mist and its installation.

Keywords:

Nebulized Water, Losses in Infrastructure and Fire Systems. 